Supplement of The Cryosphere, 15, 1383-1397, 2021

https://doi.org/10.5194/tc-15-1383-2021-supplement

(C) Author(s) 2021. CC BY 4.0 License.

(c) (1)

Supplement of

\title{
Giant dust particles at Nevado Illimani: a proxy of summertime deep convection over the Bolivian Altiplano
}

Filipe G. L. Lindau et al.

Correspondence to: Filipe G. L. Lindau (filipelindau@ hotmail.com)

The copyright of individual parts of the supplement might differ from the article licence. 


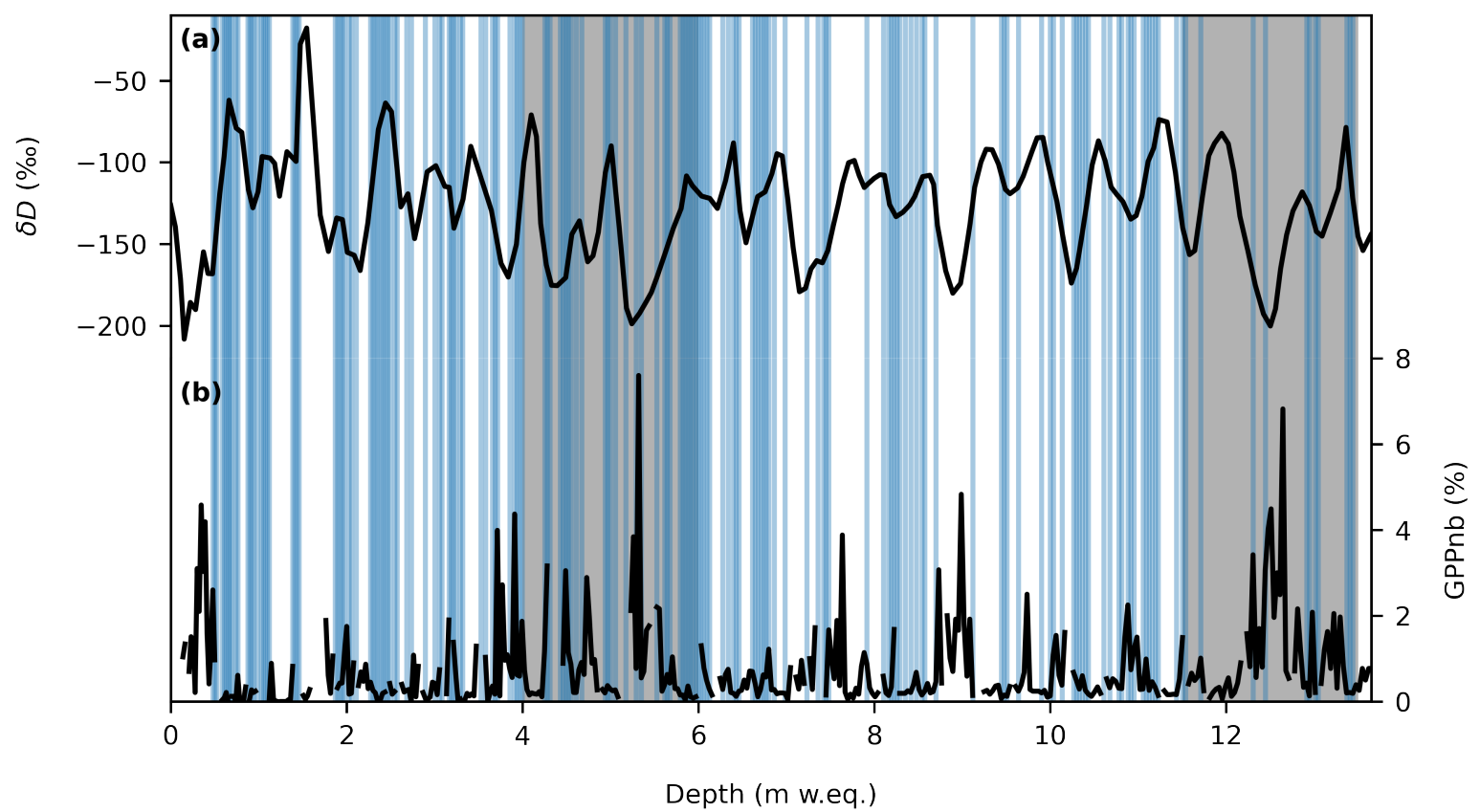

Figure S1. Ice and/or crust layers recorded in the Illimani's firn core (blue vertical lines) along with the records of $\delta \mathrm{D}$ (a) and GPPnb (b). The gray area in the depth range 4-6 mw.eq. features more ice/crust layers than the gray area within the 11.5-13.5 mw.eq. interval. However, both the variability in (a) and (b) show no evidence of signal change due to the occurrence of these layers. 
Table S1. Sections of the Nevado Illimani firn core analysed for elemental (N1 to N10) and mineralogical (R1 to R4) composition.

\begin{tabular}{|c|c|c|c|c|c|c|c|c|c|c|c|c|c|c|}
\hline Sample & N1 & $\mathrm{N} 2$ & N3 & N4 & N5 & N6 & N7 & N8 & N9 & N10 & $\mathrm{R} 1$ & $\mathrm{R} 2$ & R3 & $\mathrm{R} 4$ \\
\hline Top depth (m) & 1.67 & 2.31 & 5.57 & 8.67 & 10.56 & 13.60 & 13.69 & 19.10 & 20.29 & 21.41 & 5.79 & 5.91 & 9.80 & 10.00 \\
\hline Bottom depth (m) & 2.31 & 3.78 & 7.02 & 8.97 & 11.66 & 13.69 & 14.78 & 19.25 & 20.48 & 22.50 & 5.82 & 6.00 & 9.84 & 10.17 \\
\hline
\end{tabular}

Table S2. Average elemental concentrations measured by Instrumental Neutron Activation Analysis, and the procedural errors and detection limits (DL). For comparison, we also present mean concentrations of dust particles from high elevation ice cores in the Alps (Thevenon et al., 2009) and in Tibetan plateau (Wu et al., 2009).

\begin{tabular}{cccccccccc}
\hline Element & $\mathrm{Ce}$ & $\mathrm{Cs}$ & $\mathrm{Eu}$ & $\mathrm{Hf}$ & $\mathrm{La}$ & $\mathrm{Sc}$ & $\mathrm{Sm}$ & $\mathrm{Th}$ & $\mathrm{Yb}$ \\
\hline Concentration (ppm) & 89.8 & 21.1 & 1.6 & 13.1 & 40.8 & 13.7 & 8.14 & 17.6 & 2.71 \\
Error (\%) & 7.24 & 13.8 & 6.65 & 9.97 & 4.05 & 3.97 & 7.27 & 7.16 & 10.8 \\
DL (ppm) & 7 & 4 & 0.8 & 3 & 0.5 & 0.3 & 0.1 & 4 & 0.1 \\
\hline Colle Gnifetti ice core, Alps (ppm) & 1.76 & 1.20 & 1.78 & 5.24 & 2.74 & 18.6 & 4.63 & 3.49 & 6.25 \\
Dunde ice core, Tibetan Plateau (ppm) & 65.7 & & 1.09 & 4.04 & 32.6 & & 5.51 & 14.3 & 2.93 \\
\hline
\end{tabular}


Table S3. Mineral phases and relative abundance (number of grains, \%) in Illimani firn from different time periods.

\begin{tabular}{|c|c|c|c|c|}
\hline & $\mathrm{R} 1$ & $\mathrm{R} 2$ & $\mathrm{R} 3$ & $\mathrm{R} 4$ \\
\hline Year & 2010 & 2009-10 & 2004-05 & 2004 \\
\hline Season & dry & wet & wet & dry \\
\hline \multirow[t]{2}{*}{ Particles analyzed } & 167 & 161 & 138 & 167 \\
\hline & $\mathrm{N}(\%)$ & $\mathrm{N}(\%)$ & $\mathrm{N}(\%)$ & $\mathrm{N}(\%)$ \\
\hline Quartz and polymorphs & 31.7 & 21.7 & 13.8 & 23.4 \\
\hline K-feldspars & 7.2 & 10.6 & 5.1 & 11.4 \\
\hline Plagioclases & 10.8 & 9.3 & 7.2 & 18.6 \\
\hline Muscovite-illite-smectite & 23.4 & 29.2 & 59.4 & 19.2 \\
\hline Other phyllosilicates & 0.6 & 1.2 & 0 & 1.2 \\
\hline Clay minerals (kaolinite) & 0.6 & 1.2 & 0 & 3 \\
\hline Clinopyroxenes & 0 & 0.6 & 0 & 0 \\
\hline Orthopyroxenes & 0 & 0 & 0 & 0 \\
\hline Amphiboles & 0 & 0 & 0 & 0 \\
\hline Rutile & 2.4 & 2.5 & 0 & 0.6 \\
\hline Anatase & 4.2 & 6.8 & 4.3 & 2.4 \\
\hline Hematite & 9 & 8.7 & 7.2 & 14.4 \\
\hline Goethite & 8.4 & 5.6 & 1.4 & 3 \\
\hline Carbonates & 0 & 1.2 & 0 & 0 \\
\hline Epidotes & 0 & 0 & 0 & 0 \\
\hline Titanite & 0 & 0 & 0 & 0 \\
\hline Tourmaline & 1.2 & 0 & 0.7 & 0.6 \\
\hline Other heavy minerals & 0.6 & 1.2 & 0.7 & 1.2 \\
\hline Other light minerals & 0 & 0 & 0 & 1.2 \\
\hline Total & 100 & 100 & 100 & 100 \\
\hline
\end{tabular}



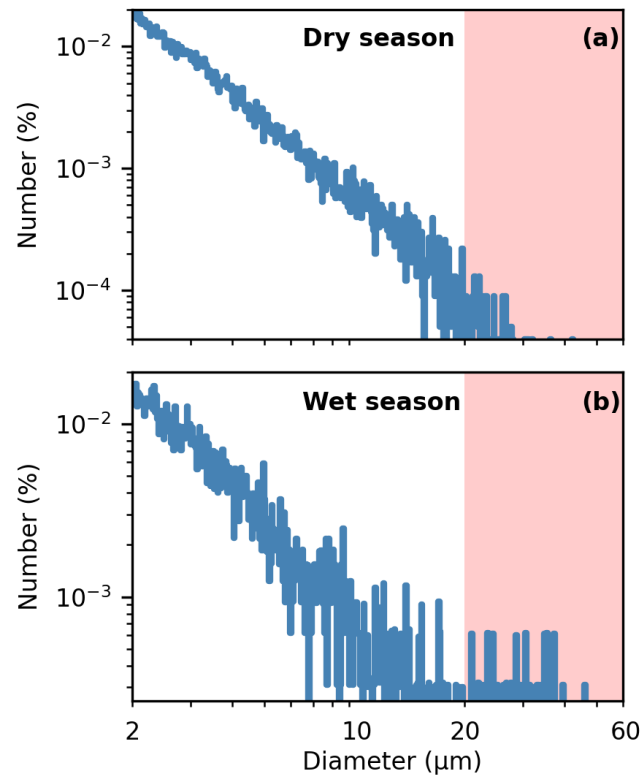

Figure S2. The number size distribuition of a typical sample from the (a) dry and (b) wet season. Red areas highlight the giant particles (between 20 and $60 \mu \mathrm{m}$ ) 


\section{References}

Thevenon, F., Anselmetti, F. S., Bernasconi, S. M., and Schwikowski, M.: Mineral dust and elemental black carbon records from an Alpine ice core (Colle Gnifetti glacier) over the last millennium, Journal of Geophysical Research Atmospheres, 114, 1-11, https://doi.org/10.1029/2008JD011490, 2009.

Wu, G., Zhang, C., Gao, S., Yao, T., Tian, L., and Xia, D.: Element composition of dust from a shallow Dunde ice core, Northern China, Global and Planetary Change, 67, 186-192, https://doi.org/10.1016/j.gloplacha.2009.02.003, 2009. 